\title{
The Nucleolus in the Mouse Oocyte is Required for the Early Step of Both Female and Male Pronucleus Organization
}

\author{
Sugako OGUSHI ${ }^{1)}$ and Mitinori SAITOU ${ }^{1)}$ \\ ${ }^{1)}$ RIKEN Center for Developmental Biology, Kobe 650-0047, Japan
}

\begin{abstract}
During oocyte growth in the ovary, the nucleolus is mainly responsible for ribosome biogenesis. However, in the fully-grown oocyte, all transcription ceases, including ribosomal RNA synthesis, and the nucleolus adopts a specific monotonous fibrillar morphology without chromatin. The function of this inactive nucleolus in oocytes and embryos is still unknown. We previously reported that the embryo lacking an inactive nucleolus failed to develop past the first few cleavages, indicating the requirement of a nucleolus for preimplantation development. Here, we reinjected the nucleolus into oocytes and zygotes without nucleoli at various time points to examine the timing of the nucleolus requirement during meiosis and early embryonic development. When we put the nucleolus back into oocytes lacking a nucleolus at the germinal vesicle $(\mathrm{GV})$ stage and at second metaphase (MII), these oocytes were fertilized, formed pronuclei with nucleoli and developed to full term. When the nucleolus was reinjected at the pronucleus (PN) stage, most of the reconstructed zygotes cleaved and formed nuclei with nucleoli at the 2-cell stage, but the rate of blastocyst formation and the numbers of surviving pups were profoundly reduced. Moreover, the zygotes without nucleoli showed a disorder of higher chromatin organization not only in the female pronucleus but also, interestingly, in the male pronucleus. Thus, the critical time point when the nucleolus is required for progression of early embryonic development appears to be at the point of the early step of pronucleus organization.
\end{abstract}

Key words: Mouse, Nucleolus, Oocyte, Pronucleus organization

(J. Reprod. Dev. 56: 495-501, 2010)

T he nucleolus is the site of ribosomal RNA (rRNA) synthesis and processing and ribosomal subunit assembly [1]. During growth in the ovary, the oocyte possesses a fibrillo-granular nucleolus with active transcription and ribosome biogenesis and stockpiles the proteins and RNAs for early embryonic development. When the oocyte reaches full size, indicating that it has acquired developmental competence, all its transcription ceases, including the transcription of rRNA. Apparently, its nucleolus no longer contains DNA at this point and shows a highly compacted, fibrillar morphology [2, 3]. As the nucleolus is eliminated during spermiogenesis [4-6], the spermatozoa do not have nucleoli. After fertilization, this maternally derived, transcriptionally inactive fibrillar nucleolus appears in both male and female pronuclei and in the embryonic nuclei of each interphase until the 4- or 8-cell stage; that is, it is present until the time at which zygotic RNA polymerase I activity is fully established [7]. The nucleolus in the oocyte or zygote is sometimes called the nucleolar precursor body (NPB) or nucleolar-like body (NLB), but we will use the term nucleolus in this study for the sake of simplicity.

When we produced an embryo lacking this inactive nucleolus, the embryo failed to develop past the first few cleavages, indicating the requirement of the oocyte nucleolus for preimplantation development [8]. However, the detailed timing of the nucleolus requirement in meiosis or in early embryonic development is not fully understood. In this study, we attempted to elucidate this tim-

Received: October 14, 2009

Accepted: May 2, 2010

Published online in J-STAGE: June 1, 2010

(C)2010 by the Society for Reproduction and Development

Correspondence: S Ogushi (e-mail: ogushi@cdb.riken.jp) ing by reinjecting the nucleolus into oocytes or zygotes lacking nucleoli and examined the embryonic defects arising from oocytes lacking a nucleolus.

\section{Materials and Methods}

\section{Mouse oocyte manipulations}

All the animals were treated with appropriate care according to the RIKEN ethics guidelines. Six- to eight-week-old B6D2F1 female mice (Japan SLC, Hamamatsu, Japan) were injected with 7.5 IU of equine chorionic gonadotropin (eCG; ASKA Pharmaceutical, Tokyo, Japan). Fully-grown GV oocytes were collected $44 \mathrm{~h}$ after injection. They were released directly into HTF-Hepes medium (Nippon Medical \& Chemical Instruments, Osaka, Japan), and their cumulus cells were removed by gentle pipetting. Oocytes were then cultured for at least $30 \mathrm{~min}$ in MEM (Sigma, St. Louis, MO, USA) with $4 \mathrm{mg} / \mathrm{ml} \mathrm{BSA,} 25 \mu \mathrm{g} / \mathrm{ml}$ sodium pyruvate, $8 \mu \mathrm{g} / \mathrm{ml}$ kanamycin and $0.1 \mathrm{mg} / \mathrm{ml}$ dibutyryl cyclic AMP (Sigma) at $37 \mathrm{C}$ under an atmosphere of $5 \% \mathrm{CO}_{2}$ in air until used for nucleolus removal by micromanipulation, i.e., enucleolation [9].

The mouse oocytes at the GV stage were manipulated with a micromanipulator equipped with a PIEZO drive (PrimeTech, Tsukuba, Japan) in modified HTF-Hepes medium supplemented with $0.1 \mathrm{mg} / \mathrm{ml}$ dibutyryl cyclic AMP and $7.5 \mu \mathrm{g} / \mathrm{ml}$ cytochalasin D (Sigma). After enucleolation, oocytes were cultured in MEM with $4 \mathrm{mg} / \mathrm{ml}$ BSA, $25 \mu \mathrm{g} / \mathrm{ml}$ sodium pyruvate and $8 \mu \mathrm{g} / \mathrm{ml}$ kanamycin for in-vitro maturation. The enucleolated oocytes that had been reinjected with nucleoli at the GV stage served as controls.

For in-vitro fertilization (IVF), fresh sperm were collected from the caudal epididymides of ten to twelve-week old B6D2F1 male 
mice (Japan SLC) and pre-cultured in T6 medium [10] for $1 \mathrm{~h}$. The manipulated oocytes at MII were selected and inseminated with capacitated spermatozoa in T6 medium for 5-6 h and then cultured in KSOM medium (Millipore, Billerica, MA, USA). Fertilization rate was assessed by the presence of two pronuclei in zygotes under an inverted microscope (Olympus Optical, Tokyo, Japan) 5-6 h after insemination.

The isolated mouse nucleoli were injected into previously enucleolated oocytes at the GV stage or at MII with a micromanipulator equipped with a PIEZO drive in modified HTFHepes medium. The enucleolated oocytes with the nucleolus reinjected at the GV stage were cultured for $16 \mathrm{~h}$, and those oocytes that reached MII were selected. The enucleolated oocytes with the nucleolus reinjected at MII were further cultured for $2 \mathrm{~h}$. Both groups of MII oocytes were then subjected to IVF. The nucleoli were reinjected into the zygotes having pronuclei at $8 \mathrm{hpf}$ (hours post-fertilization) or $15 \mathrm{hpf}$ and then inseminated. All groups of nucleolus-reinjected oocytes and zygotes were further cultured for a total of 3.5 days from the time of IVF.

For embryo transfer, two-cell embryos arising from enucleolated and from nucleolus-reinjected enucleolated oocytes were transferred into the oviducts of d- 0.5 pseudopregnant ICR female mice at 8 to 12 weeks of age. Caesarian section was performed at E19.5. Surviving pups were fostered to an ICR foster mother that had given birth on either the same day or one day earlier.

\section{Immunofluorescence microscopy}

For immunofluorescence staining, zygotes were fixed and permeabilized in $2 \%$ paraformaldehyde in phosphate-buffered saline (PBS; pH 7.4) containing $0.2 \%$ Triton $\mathrm{X}-100$ for 30 min. After blocking the samples in PBS containing $1 \mathrm{mg} / \mathrm{ml}$ BSA (PBS-BSA), the oocytes (embryos) were incubated with the appropriate primary antibodies at $4 \mathrm{C}$ overnight, washed several times in PBS-BSA and incubated with secondary antibodies for $1.5 \mathrm{~h}$ at room temperature. DNA was counterstained with $14.3 \mu \mathrm{M}$ 4',6-diamidino-2-phenylindole (DAPI, Molecular Probes, Eugene, OR, USA) for $20 \mathrm{~min}$ at 4 C. The zygotses were mounted on slides in Vectashield Mounting Medium (Vector Laboratories, Burlingame, CA, USA) and evaluated under a laser scanning confocal microscope (FluoView FV1000, Olympus Optical). For detection and analysis of the centromere position in pronuclei, the zygotes were scanned in three dimensions, a $z$ series of $0.25 \mu \mathrm{m}$ slices was recorded, and the slices were combined using the Olympus FluoView software (Olympus Optical).

The following primary antibodies were used: mouse anti-B23 (1:200; Sigma), rabbit anti-H3K9me1 (1:500; Upstate, Temecula, CA, \#07-450), rabbit anti-H3K9me2 (1:500; Upstate, \#07-441), rabbit anti-H3K9me3 (1:500; Upstate, \#07-442), rabbit antiH3K27me1 (1:500; Upstate, \#07-448); rabbit anti-H3K27me2 (1:500; Upstate, \#07-452), rabbit anti-H3K27me3 (1:500; Upstate \#07-449), rabbit anti-H3K4me3 (1:500; Upstate \#07-473), rabbit anti-H3K9ac (1:500; Upstate \#06-599) and human nuclear ANAcentromere autoantibody (CREST) antiserum (1:100; CS1058, Cortex Biochem, Concord, MA, USA). The secondary antibodies were Alexa Fluor 488-labeled goat anti-rabbit IgG, Alexa Fluor 488-labeled goat anti-mouse IgG and Alexa Fluor 488-labeled goat anti-human IgG (1:400; Molecular Probes).

\section{Statistical analysis}

In this study, 15-30 oocytes were observed immunohistochemically for each group at each time point, and representative photos are shown in the figures. The results from at least three replicate experiments are summarized. The frequencies of samples were analyzed using the chi-square test. $\mathrm{P}$ values less than 0.05 were considered to indicate statistical significance.

\section{Results}

First, we examined the timing of the nucleolus requirement during meiosis and early embryonic development. We use the term enucleolation to describe the method of plucking the nucleolus out from the oocyte by micromanipulation [9]. Using enucleolation, we isolated the nucleolus and reinjected it into the enucleolated oocyte at the GV stage, at MII and at the PN stage. The enucleolated oocytes progressed to MII, were fertilized and formed pronuclei without nucleoli, and $84 \%$ of them arrested their development between the two- or four-cell stages (Table 1, Fig. 1A). The embryos that originated from enucleolated oocytes never formed nucleoli until the 2-cell stage (Fig. 2B), but some of these embryos eventually reached the 4-cell stage and formed foci that were positive for the somatic nucleolus marker, B23. However, these foci were smaller than those of nucleoli in the non-manipulated embryos (Fig. 2A). The oocytes reinjected with nucleoli at the GV stage progressed to MII, were fertilized and formed pronuclei having nucleoli, and $67 \%$ of them developed to the blastocyst stage (Table 1, Fig. 1B). When we reinjected the nucleolus into the enucleolated oocytes at MII, the oocytes were fertilized and formed pronuclei with nucleoli, and $50 \%$ of them developed to the blastocyst stage (Table 1, Fig. 1C). In the case of nucleolus reinjection into the enucleolated oocytes at the PN stage, all of the reconstructed zygotes assembled the nucleoli in both male and female pronuclei within $3 \mathrm{~h}$, cleaved and formed nuclei with nucleoli at the 2-cell stage (Fig. 2D). The total number of these embryos that reached beyond the 4-cell stage was increased compared with that of embryos derived from enucleolated oocytes, but the rate of blastocyst formation was still low (Table 1, Figs. 1D and E). The enucleolated oocytes that had been reinjected with nucleoli at the GV stage served as controls in further experiments, since those embryos showed that patterns of immunofluorescence staining and developmental potency that were similar to those in the non-manipulated embryos.

We transplanted the 2-cell embryos derived from nucleolus-reinjected enucleolated oocytes into the uteri of pseudopregnant mice (Table 2). The birth rate of the zygotes that were reinjected with nucleoli around the time of pronucleus assembly ( $8 \mathrm{hpf}$ ) or after DNA synthesis (15 hpf) was severely low, suggesting that the nucleolus reinjection into zygotes without nucleolus materials did not effectively rescue them from developmental arrest. From the experiments above, we concluded that the oocyte nucleolus is unnecessary for meiosis progression to second metaphase, but indispensable for some undetermined stage of subsequent fertilization or early embryonic development. 
Table 1. In vitro development of mouse embryos derived from nucleolus-reinjected oocytes after 3.5 day culture

\begin{tabular}{|c|c|c|c|c|c|}
\hline Type of mouse oocytes & Enucleolated & $\begin{array}{c}\text { Nucleolus reinjection } \\
\text { at GV }\end{array}$ & $\begin{array}{c}\text { Nucleolus reinjection } \\
\text { at MII }\end{array}$ & $\begin{array}{c}\text { Nucleolus reinjection } \\
\text { at hpf } 8\end{array}$ & $\begin{array}{c}\text { Nucleolus reinjection } \\
\text { at hpf } 15\end{array}$ \\
\hline Total no. of oocytes examined & 136 & 85 & 105 & 84 & 61 \\
\hline Total no. (\%) of oocytes reached to MII & 106 (78) & $65(76)$ & & & \\
\hline Total no. (\%) of MII oocytes fertilized* & $84(79)^{a}$ & $53(82)^{\mathrm{ab}}$ & $95(90)^{\mathrm{b}}$ & & \\
\hline Total no. (\%) of zygotes cleaved** & $65(77)$ & $48(91)$ & $83(87)$ & $72(86)$ & $49(80)$ \\
\hline $\begin{array}{l}\text { Total no. (\%) of zygotes arrested } \\
\text { at the } 2 \text { cell stage*** }\end{array}$ & $31(47)$ & $4(8)$ & $15(18)$ & $26(36)$ & $17(35)$ \\
\hline $\begin{array}{l}\text { Total no. (\%) of zygotes arrested } \\
\text { at the } 3-4 \text { cell stage*** }\end{array}$ & $24(37)$ & $2(4)$ & $6(7)$ & $18(25)$ & $16(33)$ \\
\hline $\begin{array}{l}\text { Total no. (\%) of zygotes arrested } \\
\text { at the 5-16 cell stage*** }\end{array}$ & $4(6)$ & $0(0)$ & $5(6)$ & $15(21)$ & $6(24)$ \\
\hline $\begin{array}{l}\text { Total no. (\%) of zygotes arrested } \\
\text { at the morula stage*** }\end{array}$ & $3(5)$ & $10(21)$ & $16(19)$ & $8(11)$ & $8(16)$ \\
\hline $\begin{array}{l}\text { Total no. (\%) of zygotes forming } \\
\text { blastocysts*** }\end{array}$ & $3(5)$ & $32(67)^{\mathrm{a}}$ & $41(49)^{\mathrm{a}}$ & $5(7)^{b}$ & $2(4)$ \\
\hline
\end{tabular}

Values with different superscripts within the same rows are significantly different $(\mathrm{P}<0.05)$. * The percentage indicates the total number of fertilized oocytes divided by that of MII oocytes. ${ }^{* *}$ The percentage indicates the total number of cleaved zygotes divided by that of fertilized oocytes. $* * *$ The percentage indicates the total number of zygotes divided by that of cleaved zygotes.
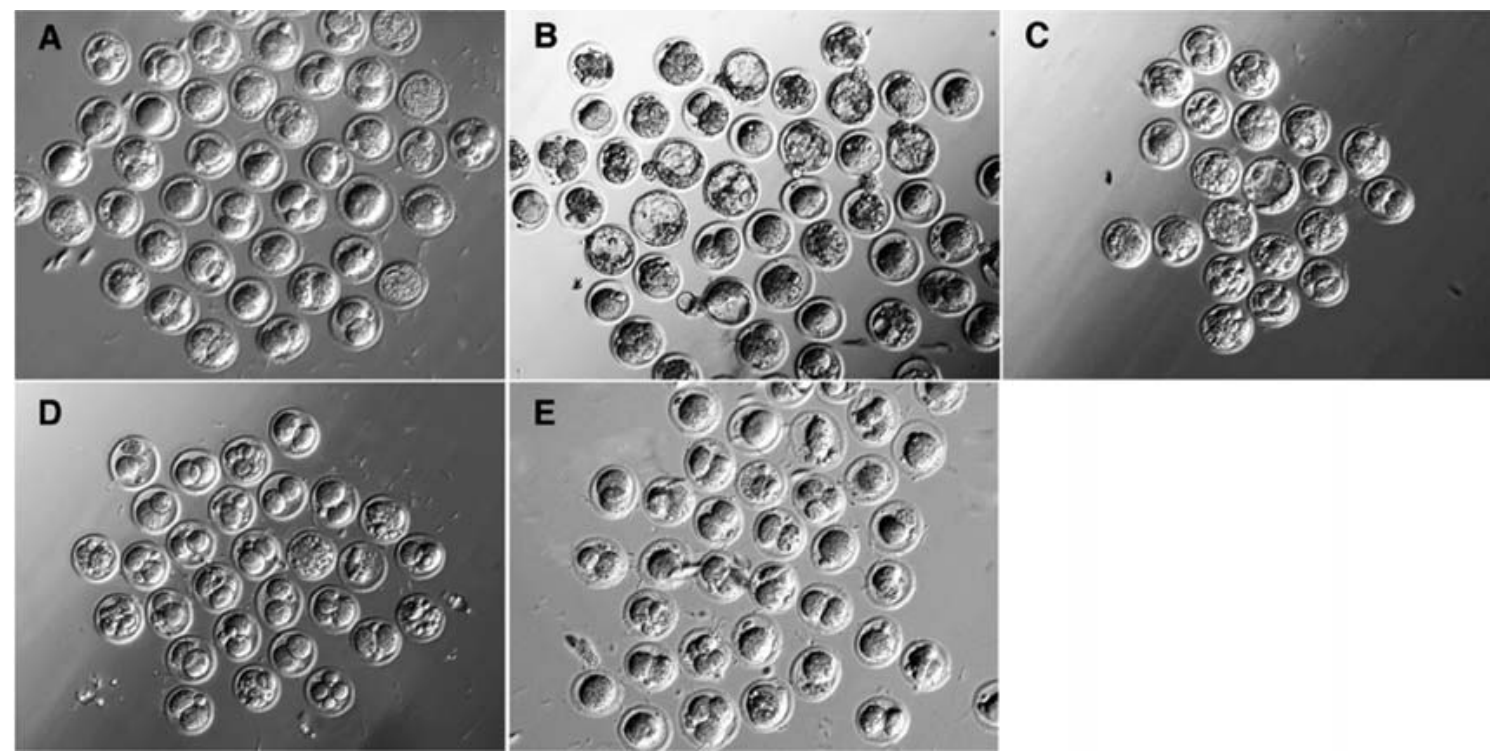

Fig. 1. Requirement of the oocyte nucleolus for the early step of pronucleus formation. The embryos originating from enucleolated oocytes (A) were arrested at the 2-4 stages or fragmented. Reinjection of nucleoli into enucleolated oocytes at the GV stage (B) or MII (C) restored the preimplantation development, whereas reinjection into the zygotes at $8 \mathrm{hpf}(\mathrm{D})$ and $15 \mathrm{hpf}(\mathrm{E})$ did not.

Nuclear architecture and chromatin organization play pivotal roles in gene regulation [11]. Because the pronucleus nucleoli move dynamically and methylated DNA [12] and centromeres [13] gather to these nucleoli, we suspect that the nucleoli in pronuclei may be involved in construction of a functional nuclear architecture. Using immunofluorescence labeling, we checked the chromatin organization and global chromatin modifications of histone N-terminals in pronuclei of zygotes having or lacking nucleoli (Fig. 3). Zygotes that arose from enucleolated oocytes that had been reinjected with nucleoli served as controls (reinjection at GV).
In the control zygotes (reinjection at GV, Fig. 3A), the female pronuclei (the left side of pronuclei in each panel) stained positive for H3K9me1/2/3, H3K27me1/2/3, H3K4me3 and H3K9ac. The male pronuclei (the right side of the pronuclei in each panel) stained positive for H3K9me1, H3K27me1/2/3 and H3K9ac, but not for H3K9me2/3 and H3K4me3 $8 \mathrm{~h}$ after insemination. There were perinucleolar heterochromatin rings in both male and female pronuclei, and most of the centromeres (CREST) localized around the nucleoli. In the zygotes lacking nucleoli (Fig. 3B), the global histone modification patterns of H3K4me3, H3K9ac, H3K9me1/2/3 

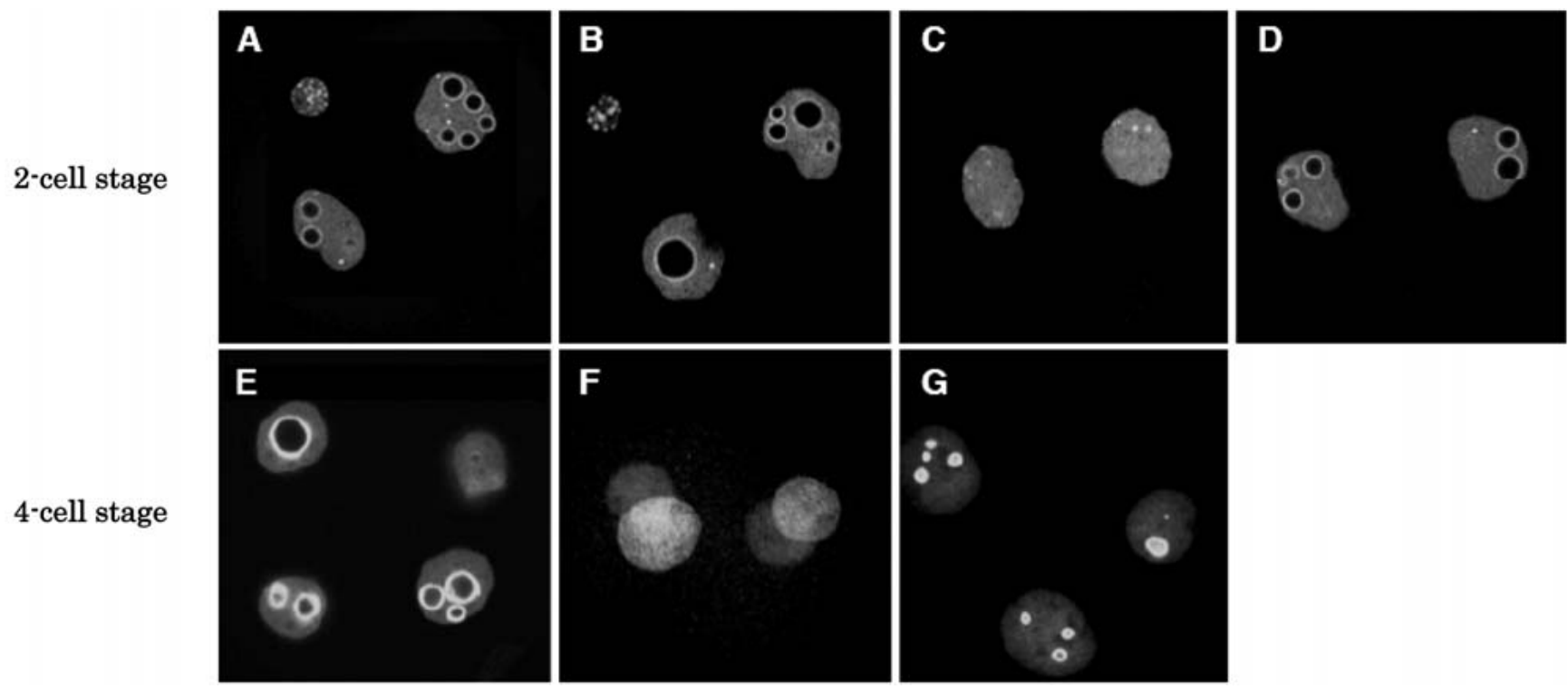

Fig. 2. The presence of nucleoli in the nuclei of embryos that arose from nucleolus-reinjected enucleolated oocytes. No nucleolus was formed in the 2cell stage embryos originating from enucleolated (C) oocytes. Nucleolus reinjection at the GV stage (B) and 8 hpf (D) restored the nucleolus formation at the 2-cell stage as in the non-manipulated embryo (A). At the 4-cell stage, the nucleoli were assembled in the embryos originating from nucleolus-reinjected enucleolated oocytes (E). Some 4-cell-stage embryos originating from enucleolated oocytes assembled small B23positive foci in their nuclei (G), but some embryos did not (F). Nucleoli in nuclei were detected by immunofluorescence labeling against B23 (in green).

Table 2. Developmental potency of mouse embryos derived from nucleolus-reinjected oocytes

\begin{tabular}{lccccc}
\hline $\begin{array}{l}\text { Type of mouse } \\
\text { oocytes }\end{array}$ & Enucleolated & $\begin{array}{c}\text { Nucleolus reinjection } \\
\text { at GV }\end{array}$ & $\begin{array}{c}\text { Nucleolus reinjection } \\
\text { at MII }\end{array}$ & $\begin{array}{c}\text { Nucleolus reinjection } \\
\text { at hpf } 8\end{array}$ & $\begin{array}{c}\text { Nucleolus reinjection } \\
\text { at hpf 15 }\end{array}$ \\
\hline $\begin{array}{l}\text { Total no. of embryos } \\
\text { transferred (reciepients) }\end{array}$ & $134(7)$ & $156(8)$ & $124(6)$ & $138(7)$ & $248(12)$ \\
$\begin{array}{l}\text { Total no. of pregnant recipients } \\
\text { Pups }\end{array}$ & 0 & 7 & 6 & 0 & 1 \\
\end{tabular}

Values with different superscripts within the same row are significantly different $(\mathrm{P}<0.05)$.

and H3K27me1/2/3 were indistinguishable from those of control zygotes (reinjection at GV). However, the zygotes that arose from enucleolated oocytes showed a disorder of higher chromatin organization $8 \mathrm{~h}$ after insemination. We observed scattered heterochromatin dots in both male and female pronuclei. In the female pronuclei, these dots colocalized with the centromeres (CREST) and were stained positive for heterochromatic modifications such as H3K9me2/3 and H3K27me1/2/3. In the male pronuclei as well, the dots were co-localized with centromeres and stained positive for H3K27me1/2/3, but these dots tended to cluster rather than scatter and were not stained positive for H3K9me2/3, demethylation of which was also observed in control zygotes (reinjection at GV). It is interesting to note that this chromatin disorganization occurs in the male pronucleus as well as in the female pronucleus (Fig. 3).
We then analyzed the nuclear localization of centromeres following insemination in zygotes that arose from enucleolated oocytes by immunofluorescence (Fig. 4). With or without nucleoli, the dot signals of centromeres (CREST) were detected in both maternal and paternal chromatin $3 \mathrm{~h}$ after insemination (Fig. 4, 3 $\mathrm{hpf}$ ). As pronucleus formation proceeded, most centromeres were localized around the nucleoli in the control zygotes (reinjection at GV, Fig. 4A); however, centromeres were equally distributed all over the pronuclei in the zygotes derived from enucleolated oocytes (Fig. 4B, 4-12 hpf). Moreover, in zygotes lacking a nucleolus, several signals of centromeres in male and female pronucleus increased in size. The number of centromere signals in each pronucleus was not affected $12 \mathrm{~h}$ after insemination (Reinjection at GV; $13.2 \pm 3.1$ (female) and 13.1 \pm 4.2 (male), Enucleolation; $14.2 \pm 2.6$ (female) and $11.1 \pm 1.8$ (male)). 
A
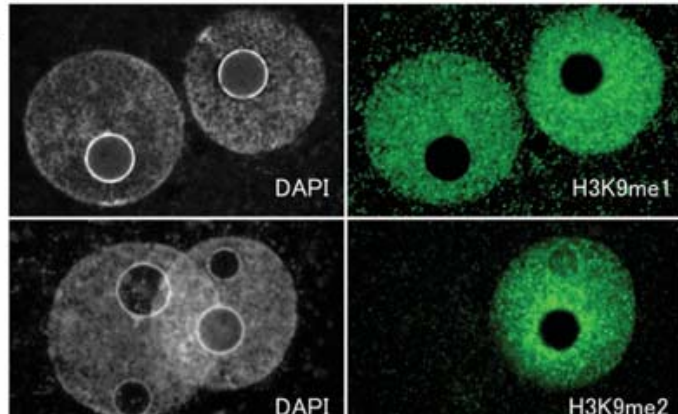

DAPI

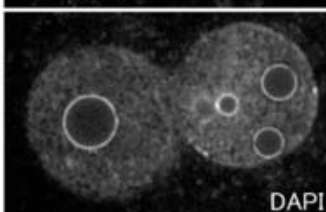

DAP
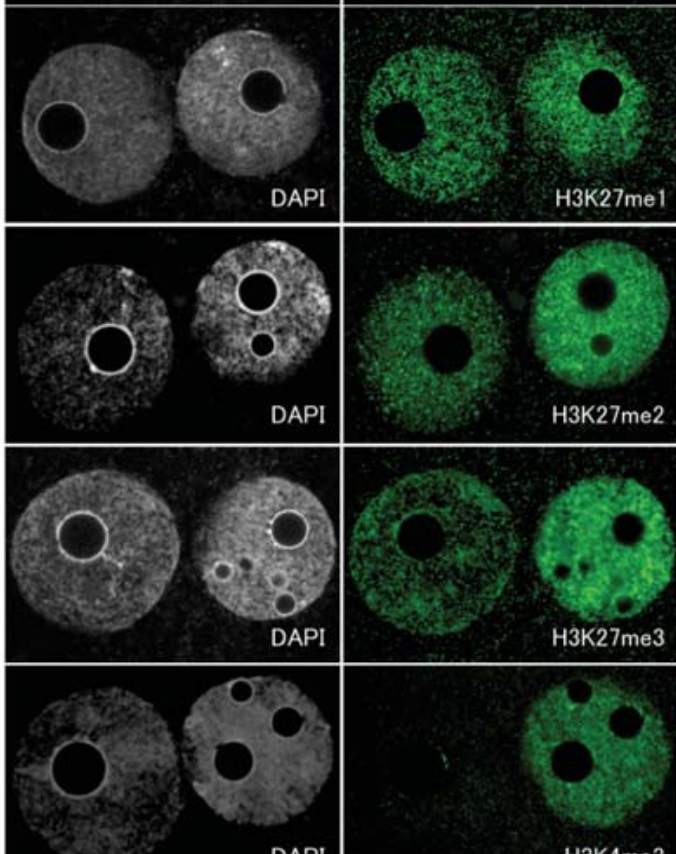

.
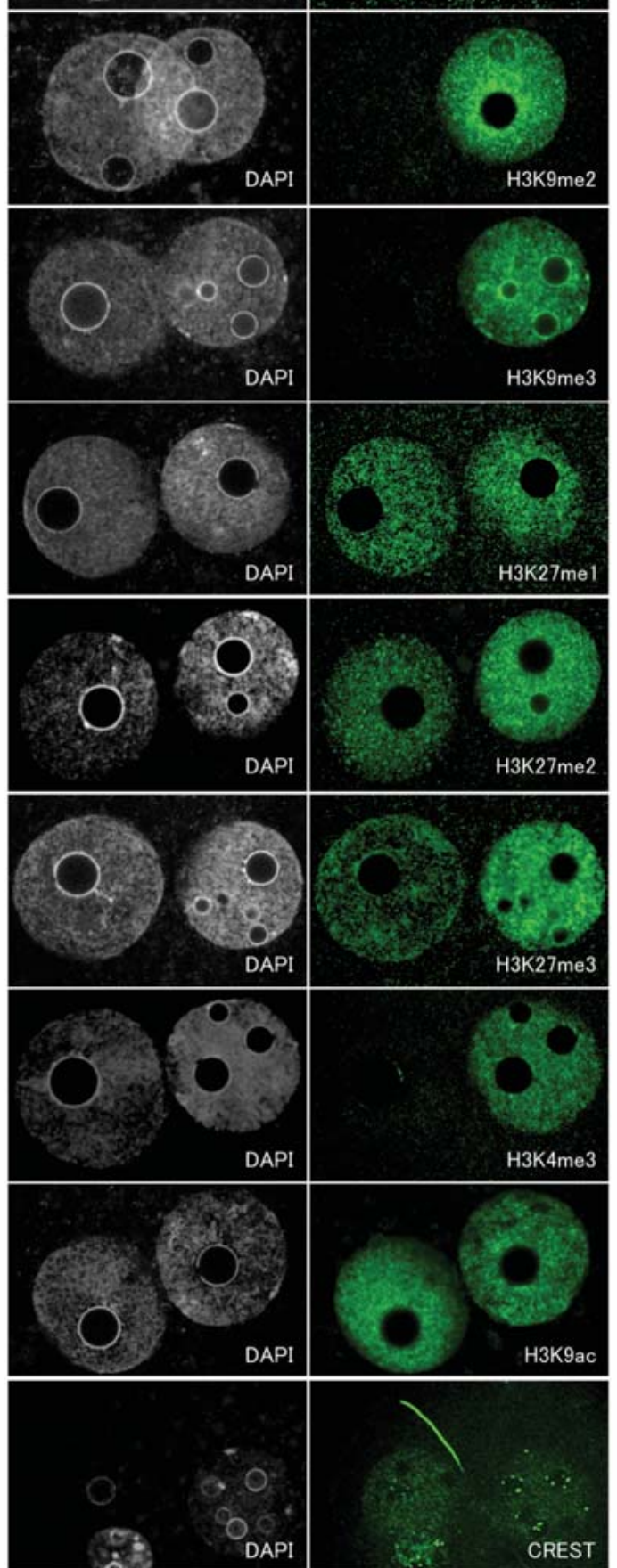

$\mathrm{B}$
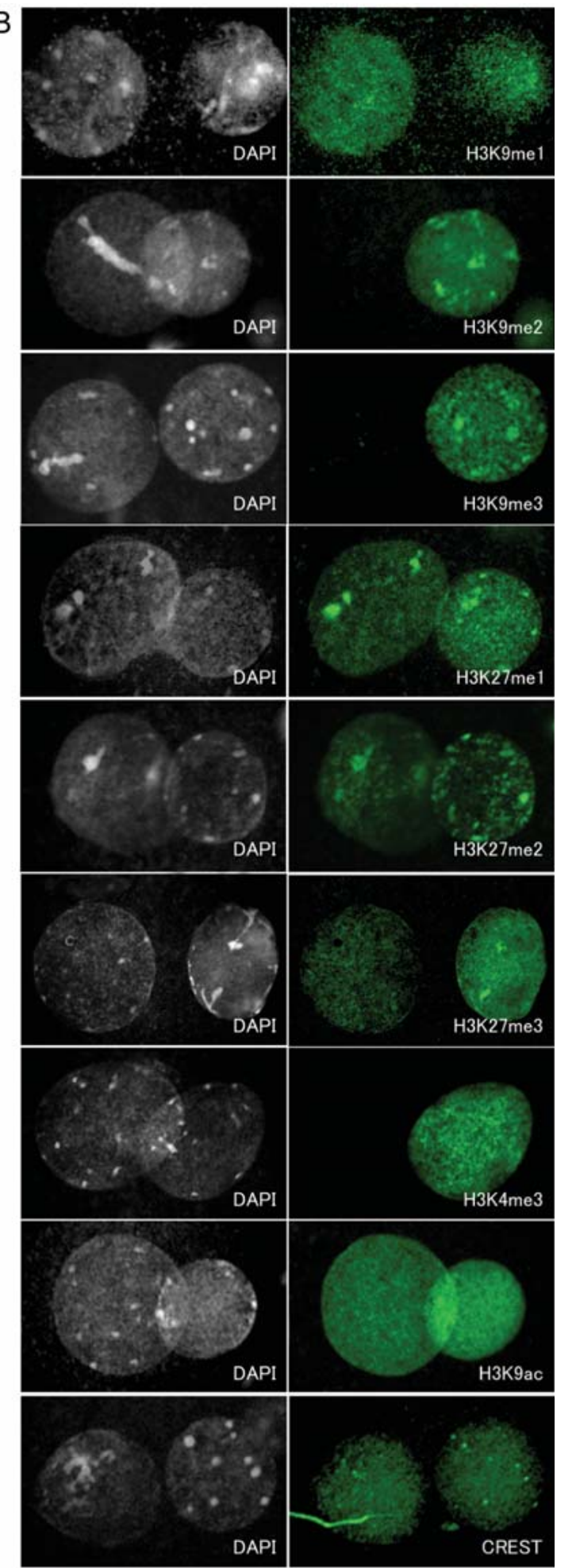

Fig. 3. The heterochromatin disorganization in enucleolated oocytes $8 \mathrm{~h}$ after insemination. Immunofluorescence labeling was performed with H3K9me1/ 2/3 (mono/di/trimethylated histone H3 at lysine 9), H3K27me1/2/3 (mono/di/trimethylated histone H3 at lysine 27), H3K4me3 (trimethylated histone H3 at lysine 4), H3K9ac (acetylated histone H3 at lysine 9), and CREST antibodies (in green). DNA was counterstained with $14.3 \mu \mathrm{M}$ 4'6-diamidino-2-phenylindole (DAPI). The zygotes derived from enucleolated oocytes (B) showed distinct heterochromatin organization in both male and female pronuclei compared with the zygotes reinjected with nucleoli at the GV stage (A). 

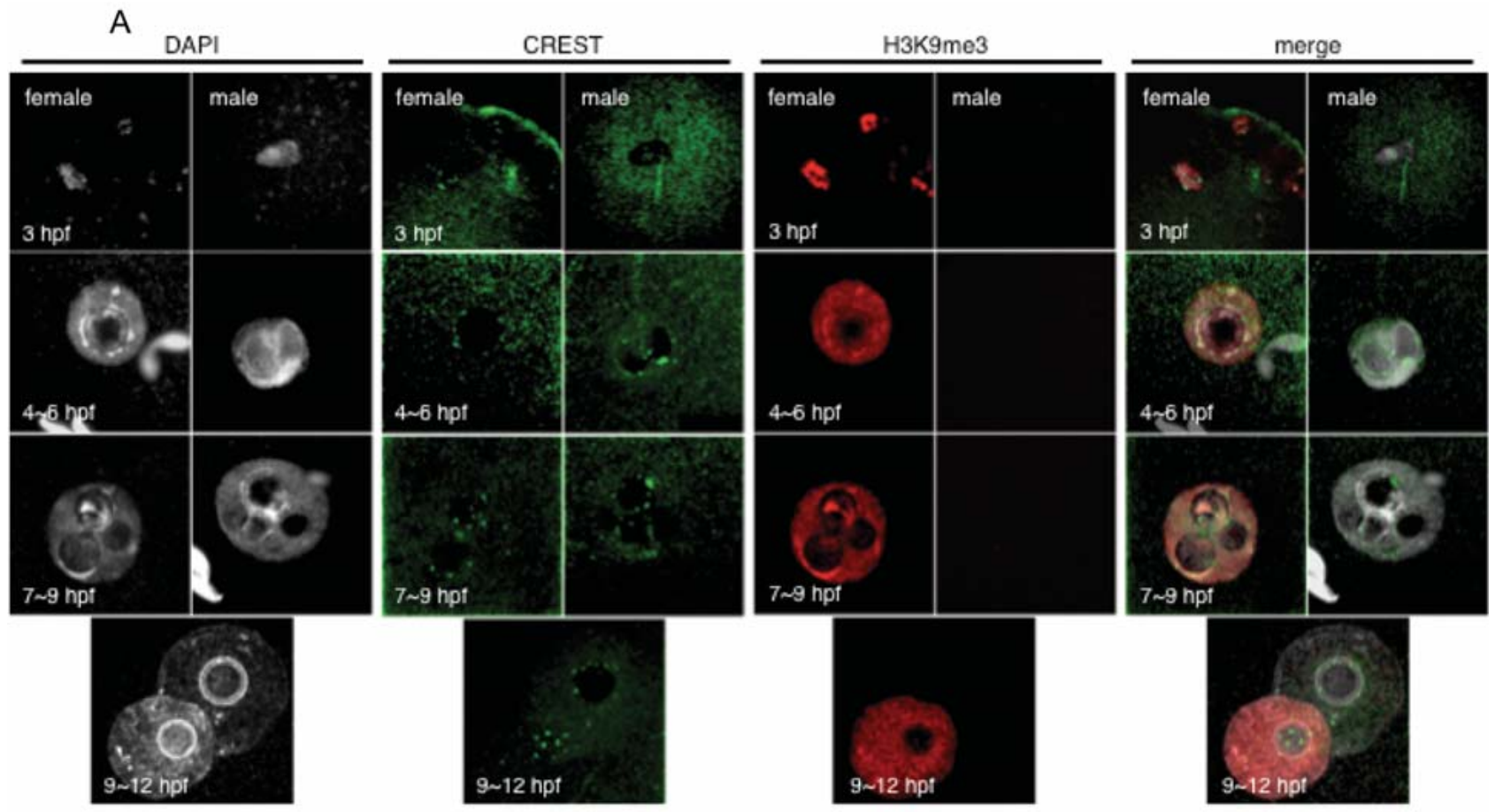

B
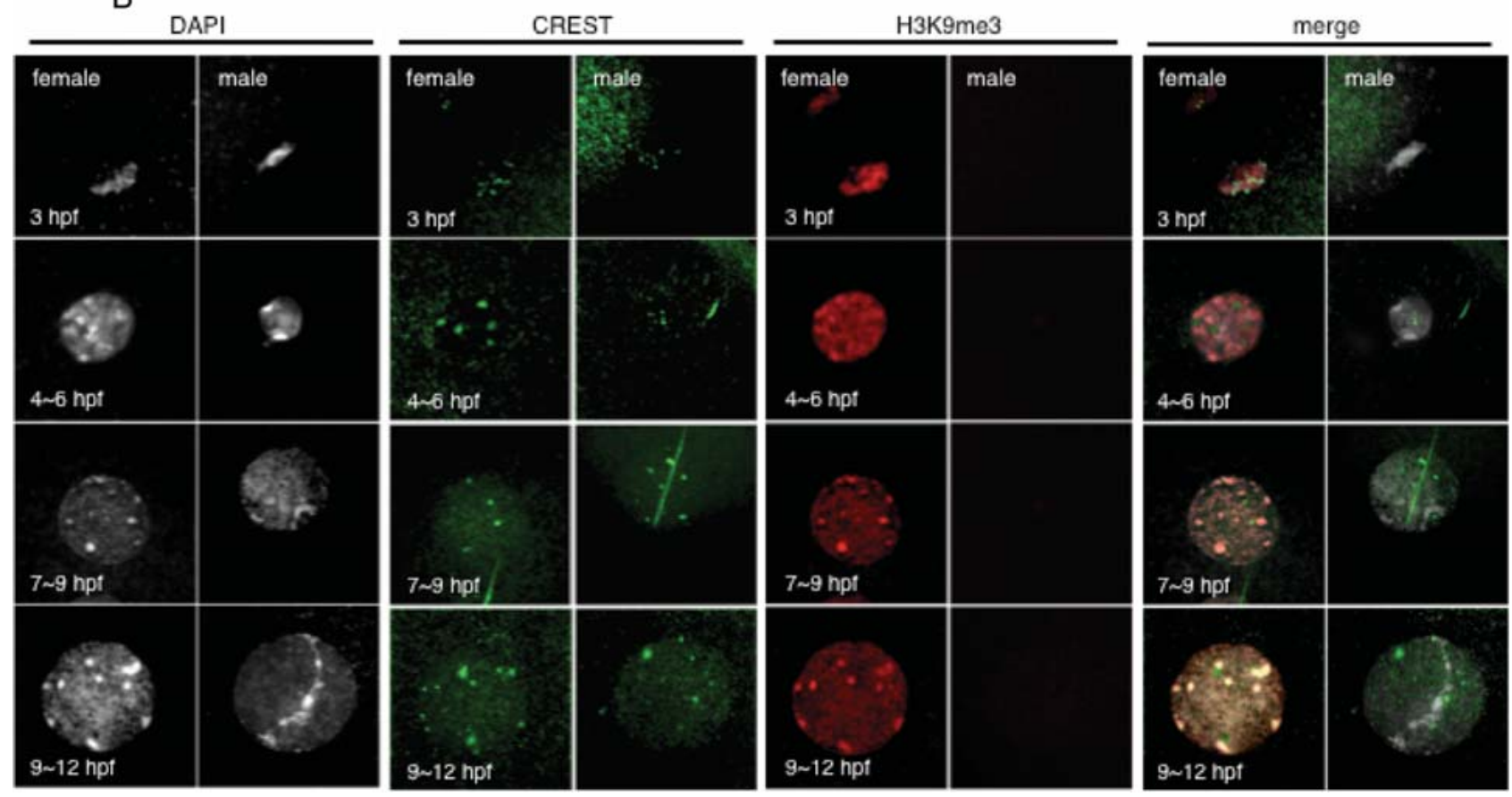

Fig. 4. Nucleolus loss alters the localization and size of centromeres in both male and female pronuclei. Immunofluorescence labeling was performed with CREST antibodies (in green), and trimethylated histone $\mathrm{H} 3$ at lysine 9 antibodies (H3K9me3) (in red). DNA was counterstained with 14.3 $\mu \mathrm{M}$ 4',6-diamidino-2-phenylindole (DAPI). Most centromeres were localized around the nucleoli in zygotes reinjected with nucleoli at the GV stage (A), but in zygotes lacking a nucleolus (B), centromeres were equally distributed all over the pronuclei, and some signals in both male and female pronuclei increased in size. 


\section{Discussion}

Following fertilization, fully differentiated gametes transform into zygotic nuclei, i.e., male- and female-pronuclei, with the acquisition of totipotency. The chromatin organization in this step displays particular features [14-16]. The centromeric heterochromatin occupies restricted spatial domains surrounding the nucleolus; however, the relation between the chromatin and nucleolus following fertilization has not been elucidated. In this study, we found that zygotes lacking a nucleolus showed global histone $\mathrm{N}$-terminal modification patterns in both male and female pronuclei that were similar to those of the control zygotes, except they were incapable of controlling the higher organization of the centromeric heterochromatin after fertilization (Figs. 3 and 4). The perinucleolar heterochromatin contains centromeric satellite DNA and ribosomal DNA repeats $[7,17]$. It is possible that the lack of the nucleolus in the zygote induces the higher chromatin disorganization of centromeric heterochromatin regions and causes the instability of the centromere and ribosomal DNA repeats. It is of note that the higher heterochromatin disorganization occurs not only in the female pronucleus but also in the male pronucleus. The nucleolus materials are only supplied from oocytes [8]; thus, the maternal nucleolus organizes both paternal and maternal centromeric heterochromatin in the pronuclei.

The nucleolus re-transplantation experiments clearly indicated that the nucleolus is involved in the transformation step from nuclei of female and male gametes to zygotic pronuclei. As enucleolated oocytes have the ability to swell the sperm head and decondense the sperm chromatin [8], the nucleolus itself is dispensable for the early step of sperm chromatin reorganization. Other than that, following sperm head swelling, the maternal and paternal chromatins recruit pronucleus materials of nucleoplasm, nuclear bodies and nuclear membrane, and expand both female and male pronuclei with heterochromatin redistribution. We suspect that nucleolus function is related to one or more of these phenomena. When the embryos that arose from the enucleolated oocytes were fixed at 24 $\mathrm{h}$ after insemination, all of them were at the first metaphase, in contrast to all the control embryos, which reached the 2-cell stage (data not shown). This cell cycle delay might be caused by lack of the oocyte nucleolus in these embryos and might be related to the centromeric heterochromatin disorganization. It remains to be elucidated, however, whether or not the centromeric heterochromatin disorganization directly causes the developmental arrest.

Some embryos derived from enucleolated oocytes reached beyond the 4-cell stage, and several of them had B23-positive foci in their nuclei (Fig. 2). B23 is involved in rRNA processing and ribosome biogenesis and is the marker for somatic cell nucleoli. B23-positive signals in embryos lacking oocyte nucleoli look similar to somatic cell type nucleoli in size and in shape [18]. Four-cell stage embryos that arise from enucleolated oocytes might reassemble the somatic type nucleoli after zygotic gene activation, which normally occurs around the 8-16-cell stage [7]. It is possible that the oocyte nucleolus suppresses the formation of a somatic cell type nucleolus during the first several cleavages.

\section{Acknowledgments}

SO is a fellow in the Special Postdoctoral Researcher Program of RIKEN. This study was supported in part by an FY2008 DRI Research Grant from RIKEN and a Grant-in-Aid for Young Scientists from the Ministry of Education, Culture, Sports, Science and Technology of Japan.

\section{References}

1. Olson MOJ. Introduction. In: Olson MOJ (ed.), The Nucleolus. New York: Landes Bioscience/ Kluwer Academict; 2004: 1-9.

2. Chouinard LA. A light- and electron-microscope study of the nucleolus during growth of the oocyte in the prepubertal mouse. J Cell Sci 1971; 9: 637-663.

3. Crozet N. Nucleolar structure and RNA synthesis in mammalian oocytes. Reprod Fertil Suppl 1989; 38: 9-16.

4. Monesi V. Synthetic activities during spermatogenesis in the mouse RNA and protein. Exp Cell Res 1965; 39: 197-224.

5. Schultz MC, Leblond CP. Nucleolar structure and synthetic activity during meiotic prophase and spermiogenesis in the rat. Am J Anat 1990; 189: 1-10.

6. Takeuchi IK, Takeuchi YK. Ethanol-phosphotungstic acid and bismuth staining of spermatid nucleoli in mouse spermiogenesis. J Struct Biol 1990; 103: 104-112.

7. Zatsepina O, Baly C, Chebrout M, Debey $\mathbf{P}$. The step-wise assembly of a functional nucleolus in preimplantation mouse embryos involves the cajal (coiled) body. Dev Biol 2003; 253: 66-83.

8. Ogushi S, Palmieri C, Fulka H, Saitou M, Miyano T, Fulka J Jr. The maternal nucleolus is essential for early embryonic development in mammals. Science 2008; 319: 613616

9. Fulka J, Jr, Moor RM, Loi P, Fulka J. Enucleolation of porcine oocytes. Theriogenology 2003; 59: 1879-1885.

10. Quinn P, Barros C, Whittingham DG. Preservation of hamster oocytes to assay the fertilizing capacity of human spermatozoa. J Reprod Fertil 1982; 66: 161-168.

11. Taddei A, Hediger F, Neumann FR, Gasser SM. The function of nuclear architecture a genetic approach. Annu Rev Genet 2004; 38: 305-345.

12. Yamazaki T, Yamagata $K$, Baba T. Time-lapse and retrospective analysis of DNA methylation in mouse preimplantation embryos by live cell imaging. Dev Biol 2007; 304: 409-419.

13. Probst AV, Santos F, Reik W, Almouzni G, Dean W. Structural differences in centromeric heterochromatin are spatially reconciled on fertilisation in the mouse zygote. Chromosoma 2007; 116: 403-415.

14. Santos F, Peters AH, Otte AP, Reik W, Dean W. Dynamic chromatin modifications characterise the first cell cycle in mouse embryos. Dev Biol 2005; 280: 225-236.

15. Morgan HD, Santos F, Green K, Dean W, Reik W. Epigenetic reprogramming in mammals. Hum Mol Genet 2005; 14: R47-58.

16. Longo F, Garagna S, Merico V, Orlandini G, Gatti R, Scandroglio R, Redi CA, Zuccotti M. Nuclear localization of NORs and centromeres in mouse oocytes during folliculogenesis. Mol Reprod Dev 2003; 66: 279-290.

17. Romanova L, Korobova F, Noniashvilli E, Dyban A, Zatsepina O. High resolution mapping of ribosomal DNA in early mouse embryos by fluorescence in situ hybridization. Biol Reprod 2006; 74: 807-815.

18. Amin MA, Matsunaga S, Uchiyama S, Fukui K. Depletion of nucleophosmin leads to distortion of nucleolar and nuclear structures in HeLa cells. Biochem J 2008; 415: 345351 